\title{
LAS WEBS DE LAS COMUNIDADES AUTÓNOMAS. Imagen corporativa y servicios
}

Fernando Sabés Turmo

Profesor del Master Oficial Marketing y Comunicación Corporativa de la Universidad San Jorge.

\section{Resumen}

Este artículo ofrece un análisis de los portales web institucionales de las Comunidades Autónomas españolas, unas páginas en las que la comunicación corporativa se une a la necesidad de prestar servicios a los ciudadanos. Además, no hay que olvidar que la aprobación de la Ley 11/2007, de 22 de junio, de acceso electrónico de los ciudadanos a los Servicios Públicos, supone una auténtica revolución en cuanto a la oferta de servicios desde estos portales. Estas páginas se convierten en la puerta de entrada de los ciudadanos a las Instituciones y suponen un elemento vital en la imagen que desde ellas se quiere proyectar. De todos modos, estamos ya en una fase mucho más avanzada, en la que la imagen corporativa ha de ir de la mano con los servicios de e-Administración que se deben ir implementando en todas las Instituciones públicas.

\section{Palabras clave}

Web - Comunidad Autónoma - e-Administración - imagen corporativa - servicios - ciudadanos.

\section{Abstract}

This paper offers an analysis of institutional webs in Spanish counties, where corporate image meets the needs of citizenship services. Besides, the passing of the 11/2007 Bill of citizens e-access to Public Services, on 22nd June, entails a clear revolution as far as these webs are concerned. For they 
become the door for citizens to access institutions, as well as a vital tool for image projection. All in all, corporate image goes hand in hand together with public e-admin services.

\section{Key words}

Web - County - e-Government - corporate image - services - citizens.

\section{Introducción}

Los portales web de las administraciones públicas se han convertido en la puerta de entrada cada vez más común de los ciudadanos a la información y a los servicios que prestan estas Instituciones. Supone, por un lado, un elemento vital en la imagen corporativa, pero a la vez también ha de combinar la información ofrecida en la web con la prestación de servicios a los ciudadanos.

En esta dicotomía se encuentran los portales de las Comunidades Autónomas, entes prestadores de servicios que deben estar próximos a los ciudadanos y también al resto de administraciones. La necesidad de que estas webs mantengan la imagen corporativa de la Institución es clave para conseguir la identificación de los ciudadanos, pero a la vez también deben ir incorporando cada vez más servicios, encaminados a la puesta en marcha real de la e-Administración. En este sentido, se ven obligados con la aprobación de la Ley 11/2007, de 22 de junio, de acceso electrónico de los ciudadanos a los Servicios Públicos, que debe suponer una auténtica revolución tanto para las administraciones como también para los ciudadanos que conocerán una nueva forma de relacionarse con el ámbito público.

La Ley 11/2007, de 22 de junio, de acceso electrónico de los ciudadanos a los Servicios Públicos, es una apuesta del Gobierno de España por establecer el marco de incorporación de una verdadera Administración on line en el Estado. Todo ello aprovechando la generalización de las nuevas tecnologías de la información y de la comunicación en la sociedad.

Con esta norma, se pretende cambiar completamente la Administración española, adaptándola a los nuevos tiempos y sobre todo permitiéndole eliminar las barreras de espacio y de tiempo que sí marcan el día a día del ámbito público en la actualidad. 
Esta Ley viene precedida de otras normas que ya fueron encaminando a la Administración española hacia la e-Administración, como es el caso de la Ley 30/1992 de Régimen Jurídico de las Administraciones Públicas y del Procedimiento Administrativo Común (LRJAP-PAC), que impulsó la aplicación de las técnicas y medios electrónicos, informáticos y telemáticos por parte de la Administración para desarrollar su actividad y de permitir a los ciudadanos relacionarse con las Administraciones cuando fuese compatible con los medios técnicos de que dispongan. Esa previsión, junto con la de la informatización de registros y archivos del artículo 38 de la misma Ley en su versión originaria y, especialmente, en la redacción que le dio la Ley 24/2001 de 27 de diciembre al incorporar el establecimiento de registros telemáticos para la recepción o salida de solicitudes, escritos y comunicaciones por medios telemáticos, abría el paso a la utilización de estos medios para relacionarse con la Administración. Simultáneamente, la misma Ley 24/2001 modificó el artículo 59 permitiendo la notificación por medios telemáticos si el interesado hubiera señalado dicho medio como preferente o consentido expresamente. En el mismo sentido destacan las modificaciones realizadas en la Ley General Tributaria para impulsar también las notificaciones telemáticas así como el artículo 96 de la nueva Ley General Tributaria de 2003 que prevé expresamente la actuación administrativa automatizada o la imagen electrónica de los documentos.

Pero la principal novedad de la Ley 11/2007, de 22 de junio, de acceso electrónico de los ciudadanos a los Servicios Públicos, es que garantiza su relación con las Administraciones Públicas por medios electrónicos como un derecho para los usuarios y como una obligación para las Administraciones.

\section{Objetivos}

El objetivo que se plantea en este artículo es ofrecer una visión actual del desarrollo de la administración electrónica en las Comunidades Autónomas españolas y su relación directa con la imagen que proyectan las Instituciones a los ciudadanos. De esta forma, se pretende realizar un informe vinculado al análisis crítico de estos portales webs y los servicios que prestan desde estos soportes a los ciudadanos.

Este estudio va en consonancia con la reciente aprobación de diferente legislación tanto a nivel estatal como en el marco europeo vinculada a la e-Administración. Sin embargo, se puede constatar que la aplicación de esta normativa es todavía insuficiente cuando se abordan los casos particulares de estas páginas web institucionales. 
Por decirlo de alguna forma, los fundamentos teóricos y legales se desarrollan con velocidad diferente a su aplicación. Sin embargo, esto no se produce porque la tecnología no permita su implementación de forma inminente, sino más bien porque existe una falta notable de recursos para poder aplicar los planteamientos diseñados previamente.

Pero a la vez también, la tan esperada e-Administración abre de nuevo la puerta a que se pueda producir en España una nueva brecha digital no tanto por la falta de alfabetización en el uso (que también se puede constatar en algunos sectores de la población), sino por las deficiencias en infraestructuras que se detectan en el territorio español y sobre todo en las áreas donde por la baja densidad de población la empresa privada mira hacia otro lado y no invierte en tecnología. La liberalización total del sector ha conllevado a que se incrementen estas diferencias entre unas zonas y otras y es aquí donde las administraciones están intentado hacer frente a esta brecha digital con planes directores de desarrollo de la Sociedad de la Información que, sin embargo, en demasiadas ocasiones llegan tarde o son insuficientes por la constante evolución tecnológica y sobre todo por las variaciones de las necesidades tanto de los productores de contenidos como de los consumidores.

Que las páginas web institucionales sean la puerta de entrada del ciudadano supone cambiar completamente el sistema de relación tradicional de la Administración con las personas. Es aquí donde empieza a ser fundamental la comunicación y la imagen corporativa y, por tanto, el papel que los profesionales de la comunicación han de jugar en el desarrollo de estas nuevas herramientas.

No se puede pensar ya en la imagen de una Comunidad Autónoma ni de cualquier otra Administración sin un buen trabajo corporativo en la web. Por tanto, donde los contenidos deberán encajar en este planteamiento, siendo dinámicos, actualizados, accesibles y útiles.

“Uno puede esperar que el uso de los nuevos medios de Internet también puede afectar al sistema de Gobierno local por los cambios que se están dando en las formas de comunicación política” (Olsen, Rieper y Torpe, 2006:301).

El rechazo que las nuevas tecnologías de la comunicación y de la información todavía genera en la población es cada vez menor, aunque persiste en buena parte de la población. Esta situación condiciona, y mucho, el desarrollo a corto y medio plazo de una relación on line del ciudadano con la Administración, pese a los esfuerzos en materia de seguridad que se están realizando con la aparición, por ejemplo, del DNI electrónico.

Este hecho conlleva la necesidad de un trabajo exhaustivo de identificación de los usuarios con estos portales web institucionales, que además de ofrecer de la forma más sencilla posible los servicios 
permitan al ciudadano una relación de confianza a través de una imagen corporativa coherente y bien desarrollada.

Por todo ello, en el trabajo pretendemos ofrecer una visión actual de los portales de las 17 Comunidades Autónomas y de las Ciudades Autónomas (Ceuta y Melilla) a través del análisis de los servicios que se ofrecen a los ciudadanos y los contenidos informativos que se incorporan.

\section{Metodología}

El trabajo se centrará en el estudio de las páginas webs de las 17 Comunidades Autónomas y de las dos ciudades autónomas (Ceuta y Melilla) consultadas durante los meses de noviembre y diciembre de 2007.

El análisis que proponemos será básicamente cuantitativo. Se evaluaran la presencia de variables de carácter formal, es decir no tanto qué se dice sino cómo se dice (Igartua, 2006). Se trata pues de un análisis de contenido cuya característica básica según Berelson (1971) es una técnica para estudiar y analizar la información de una manera objetiva, sistemática y cuantitativa. Krippendorff (1990) extiende esta definición entendiendo que el análisis de contenido es una técnica de investigación que permite realizar inferencias válidas y confiables de datos con respecto a su contexto.

Para Hernández Sampieri (2004:412) “el análisis de contenido puede aplicarse virtualmente a cualquier forma de comunicación”, entre ellas a las páginas web en tanto que soporte comunicativo. Para ello, se abordarán las webs en tres niveles diferenciados: acceso electrónico de los ciudadanos a los servicios públicos, tratamiento de los contenidos y aspectos de diseño e imagen.

El análisis de páginas WEBs introduce nuevos aspectos comunicativos diferenciales respecto de prensa, radio y TV. Entre ellos destaca el concepto de usabilidad (Manchón, 2002) que incorpora las dimensiones de navegación y diseño (AUNA, 2004) en la que se centra el análisis.

El primero de ellos, el de acceso electrónico de los ciudadanos a los servicios públicos, está directamente relacionado con la Ley 11/2007 de 22 de junio, y se analizarán aspectos como el ejercicio de los derechos y cumplimiento de deberes a través de medios electrónicos, el acceso a la información y al procedimiento administrativo por medios telemáticos o la existencia de elementos de confianza en el uso de estos medios, entre otros. 
En el tratamiento de los contenidos informativos, se pretende hacer un repaso a la incorporación de encuestas, notas informativas, audios, vídeos, idiomas utilizados, formularios, versión para discapacitados.

El tercer bloque a estudiar hace referencia a elementos de imagen y diseño enlazados con la proyección corporativa del portal.

La herramienta básica de trabajo ha sido la ficha que se reproduce a continuación, que ha permitido recabar datos de cada una de las webs institucionales a lo largo del mes de noviembre.

FICHA DE ANÁLISIS

\section{Acceso electrónico de los ciudadanos a los servicios públicos}

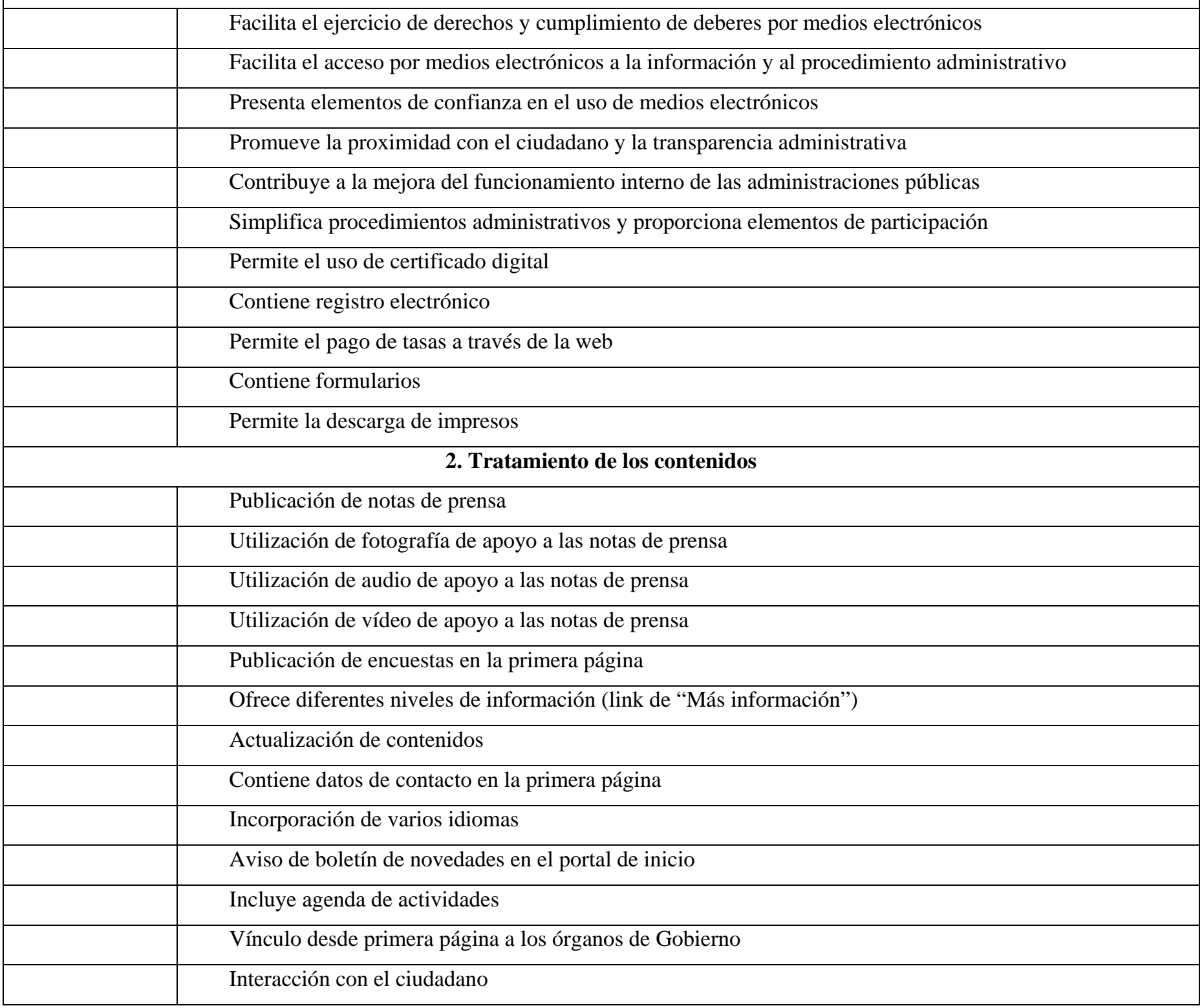




\begin{tabular}{|l|l|}
\hline & Facilidad de navegación en el dominio \\
\hline & Nivel de accesibilidad mínimo \\
\hline & Pantalla de inicio inferior a 34 KB (permite descarga rápida independientemente de la conexión) \\
\hline & Incorpora mapa web \\
\hline & Ventanas emergentes \\
\hline & Inclusión del logotipo de la Comunidad Autónoma en página de inicio \\
\hline & Incorporación de otros elementos identificativos de la Comunidad Autónoma en página de inicio \\
\hline & Existencia de menús desplegables \\
\hline & Uso de flash \\
\hline & Aparecen enlaces rotos \\
\hline & Interfaz agradable \\
\hline & Visualización correcta en diferentes navegadores \\
\hline
\end{tabular}

\section{Cambios en el diseño de las webs institucionales}

Uno de los aspectos que van a marcar el desarrollo de los portales de las diferentes administraciones públicas será la facilidad que ofrezca a los ciudadanos el acceso a los servicios que éstas prestan. Con este planteamiento, se observa ya una tendencia en cuanto a la modificación del diseño de los portales web de las administraciones españolas, dejando a un lado la tradicional estructura dividida en áreas de trabajo, que suele coincidir en el caso de la Comunidades Autónomas por consejerías, a una nueva organización en la que prima una estructura dividida por perfiles. Es el caso, por ejemplo, de Andalucía, País Vasco o Cataluña que organizan su web a través de tres ítems (ciudadanos, empresas y administraciones) o los ejemplos de la Comunidad de Murcia o Asturias que pormenorizan todavía más algunas de las categorías.

“La comunicación institucional debería ser el objetivo de mínima de toda aplicación web. Pero las necesidades de eficiencia no finalizan allí. El valor añadido en servicios y contenidos es un requerimiento cada vez mayor de los públicos objetivos, siendo productos referenciales las aplicaciones de eGoverment de ayuntamientos y gobiernos en todo el mundo” (Pardo, 2006:142). 


\section{Acceso electrónico de los ciudadanos a los servicios públicos}

La Ley 11/2007 de 22 de junio de acceso electrónico de los ciudadanos a los servicios públicos garantiza una relación entre Administración y los ciudadanos similar a la que actualmente pueden tener mediante la presencia física. Así los usuarios tienen derecho a realizar sus gestiones tanto telemáticamente como físicamente.

Para garantizar esta relación on line, las Administraciones están obligadas a diseñar diferentes medios electrónicos para la prestación de estos servicios, destacando una serie de principios que deben asumir todas las plataformas de las Instituciones como es el caso de la usabilidad (que la web sea útil), accesibilidad, legalidad en cuanto al mantenimiento de las garantías jurídicas, seguridad, veracidad y autenticidad de las informaciones, neutralidad tecnológica, adaptabilidad al progreso de las técnicas y sistemas de comunicaciones electrónicas, simplicación administrativa y transparencia y publicidad del procedimiento.

Manchón (2002) considera que la usabilidad se define por los siguientes principios básicos: “Facilidad de aprendizaje: se refiere a la facilidad con la que nuevos usuarios pueden tener una interacción efectiva. Está relacionada con la predicibilidad, sintetización, familiaridad, la generalización de los conocimientos previos y la consistencia; Flexibilidad: hace referencia a la variedad de posibilidades con las que el usuario y el sistema pueden intercambiar información. También abarca la posibilidad de diálogo, la multiplicidad de vías para realizar la tarea, similitud con tareas anteriores y la optimización entre el usuario y el sistema; Robustez: es el nivel de apoyo al usuario que facilita el cumplimiento de sus objetivos. Está relacionada con la capacidad de observación del usuario, de recuperación de información y de ajuste de la tarea al usuario”.

De todos modos, a pesar de que la usabilidad es un elemento imprescindible en el diseño de portales web que presten servicios a la ciudadanía ésta no lo es todo, según Nielsen (2000).

\subsection{Algunas cifras de la e-Administración en España}

Antes de adentrarnos en el análisis de las webs de las Comunidades Autónomas consideramos conveniente reflejar algunas cifras sobre la situación actual de la Administración electrónica en España. 
El Estado se encuentra por debajo de la media según la comparativa de $e$ Europe ${ }^{1}$ de servicios que se ofrecen a los ciudadanos por parte de las Administraciones Públicas, pero, no obstante, en las prestaciones que dan a empresas está por encima. Sin embargo, hay que indicar que en esa relación entre Administración y ciudadanía, España ha recortado las distancias en relación con los países más desarrollados.

De hecho, en España, la e-Administración ha reunido en 2006 un total de 8.240.154 usuarios, según revela el estudio “España 2007. Informe anual sobre el desarrollo de la Sociedad de la Información en España” de la Fundación Orange. Entre las gestiones realizadas de forma telemática priman las que se han llevado a cabo con la Agencia Tributaria Española. En total, se contabilizaron, por ejemplo, 2.373.164 ciudadanos que utilizaron las gestiones on line para realizar la declaración de la renta en 2006.

Por otro lado, también hay que indicar que en el caso español existe una mayor utilización de los servicios telemáticos cuando hablamos de la Administración central, mientras que tanto la regional como la local ven como merman el número de usuarios con respecto a la anterior. "Esta diferencia se debe a que hasta el momento la Administración central ofrece un catálogo más amplio de servicios electrónicos, y a que estos servicios se llevan prestando desde hace más tiempo. El tiempo es, precisamente, un factor determinante en la demanda de estos servicios, así por ejemplo, el empadronamiento y las denuncias a la policía han sido los servicios que más recientemente se han puesto a disposición del público y, por tanto, los que menor demanda tienen en la actualidad” (Fundación Orange, 2007: 234).

Las distancias entre unos territorios y otros a la hora de implantar servicios on line por parte de las diferentes Administraciones es una constante. Este hecho hace pensar también en los problemas para la homogeneización del desarrollo de la e-Administración en el ámbito autonómico y local en un futuro próximo ${ }^{2}$. Éste es uno de los grandes retos, el de la equiparación de las distintas

\footnotetext{
${ }^{1}$ La Comisión Europea realiza desde octubre de 2001 un informe de evaluación comparativa de la acción eEurope en el ámbito e-Administración. Se trata de una encuesta sobre el estado de avance en la implantación de los servicios públicos electrónicos

${ }^{2}$ El grupo de investigación Localcom, en el que participamos investigadores de seis universidades españolas (Autónoma de Barcelona, Europea de Madrid, Málaga, Alicante, Santiago de Compostela y País Vasco) ha realizado una investigación denominada Nueva tecnología de la Información y Cambio Comunicativo en las Comunidades Autónomas, un trabajo financiado por el Gobierno central desde 2003 y cuyos resultados se expusieron a finales de 2006 . El Índice Localcom, que mide los principales factores socioeconómicos, las infraestructuras tecnológicas y las pautas de consumo,
} 
administraciones, independientemente de su tamaño, al proceso de incorporación de la relación con el ciudadano de forma telemática.

Estos datos están en pleno crecimiento. La multiplicación de los servicios ofrecidos por las Administraciones, la generalización de la banda ancha y sobre todo la cada vez más confianza de los ciudadanos en las nuevas tecnologías de la información y de la comunicación hacen necesaria la apuesta definitiva por parte de las Administraciones españolas en la implantación total de la eAdministración ${ }^{3}$.

\subsection{Los servicios públicos en los portales autonómicos}

Tras realizar el estudio sobre la oferta on line de servicios públicos en las webs de las Comunidades Autónomas se constata una clara diferencia entre un grupo de Instituciones que demuestran una especial preocupación (y así la plasman con su implementación) por ofrecer un buen número de servicios a los ciudadanos telemáticamente con respecto al resto y además los plantean de forma accesible, presentan elementos de confianza suficientes para garantizar las gestiones y promueven la cercanía y la transparencia con el usuario. En este bloque hallamos las webs de Catalunya (www.gencat.net), Comunidad de Madrid (www.madrid.org) y Principado de Asturias (www.asturias.es). Las tres permiten el uso del certificado electrónico en las transacciones e incluso en la de Cataluña se puede observar como la propia Generalitat ha creado una entidad certificadora, CATCert, para las administraciones públicas catalanas, un elemento que da seguridad a los usuarios y contribuye a la mejora del funcionamiento interno de estas Instituciones.

En el segundo bloque observamos las páginas web de Comunidades que pese a realizar esfuerzos en la implantación de servicios de e-Administración, pero bien por el diseño, por la

asociadas al despliegue de la Sociedad de la Información, ofrece los resultados dispares para las comunidades de Andalucía, Aragón, Castilla La Mancha, Castilla y León, Cataluña, Comunidad Valenciana, Galicia, Madrid y País Vasco (que son las que se incorporaron al trabajo). Si la media española, según este índice es del 0,528, la Comunidad de Madrid lideraba el ranking con 0,916; seguida de Cataluña (0,754), País Vasco (0,748), Aragón (0,538), Comunidad Valenciana (0,395), Castilla y León (0,379), Galicia (0,296), Andalucía $(0,272)$ y Castilla La Mancha $(0,255)$.

${ }^{3}$ Si algún lector está interesado en profundizar en el desarrollo de la Sociedad de la Información por regiones en España, puede consultar los informes por Comunidades Autónomas que ha desarrollado el grupo LOCALCOM. 
dificultad en cuanto a la realización de estos trámites o por la falta de transparencia ${ }^{4}$ en sus transacciones muestran todavía algunas carencias: Galicia (www.xunta.es), Castilla León (www.jcyl.es), Castilla La Mancha (www.jccm.es), País Vasco (www.euskadi.net), Región de Murcia (www.carm.es), Extremadura (www.juntaex.es), Navarra (www.navarra.es), Cantabria (www.gobcantabria.es), La Rioja (www.larioja.org), Melilla (www.melilla.es) y Andalucía (www.andaluciajunta.es). Podemos destacar que los portales de Murcia, Andalucía, Navarra, País Vasco, Melilla y Castilla León tienen elementos que podrían situarlos en el primer grupo, pero presentan alguna deficiencia en cuanto al acceso de los ciudadanos a los servicios públicos y no están todavía diseñados de acuerdo a la corriente más actual de dividir los portales en los bloques de ciudadanos, empresas y administraciones.

El último de los grupos lo conforman los portales de las comunidades de Aragón (www.aragon.es), Comunidad Valenciana (www.gva.es), Canarias (www.gobcan.es), Ceuta (www.ceuta.es) y Baleares (www.caib.es). Se caracterizan, en su mayoría, por incorporar algunos trámites, aunque no con la claridad ni la transparencia de los anteriores, lo que dificulta el ejercicio de los derechos y el cumplimiento de los deberes para los ciudadanos.

\section{Gráfico 1: Servicios públicos en los portales autonómicos}

\footnotetext{
${ }^{4}$ Se entiende como principio de transparencia al ofrecimiento de la máxima difusión y publicidad de las actuaciones administrativas mediante el uso de medios electrónicos.
} 


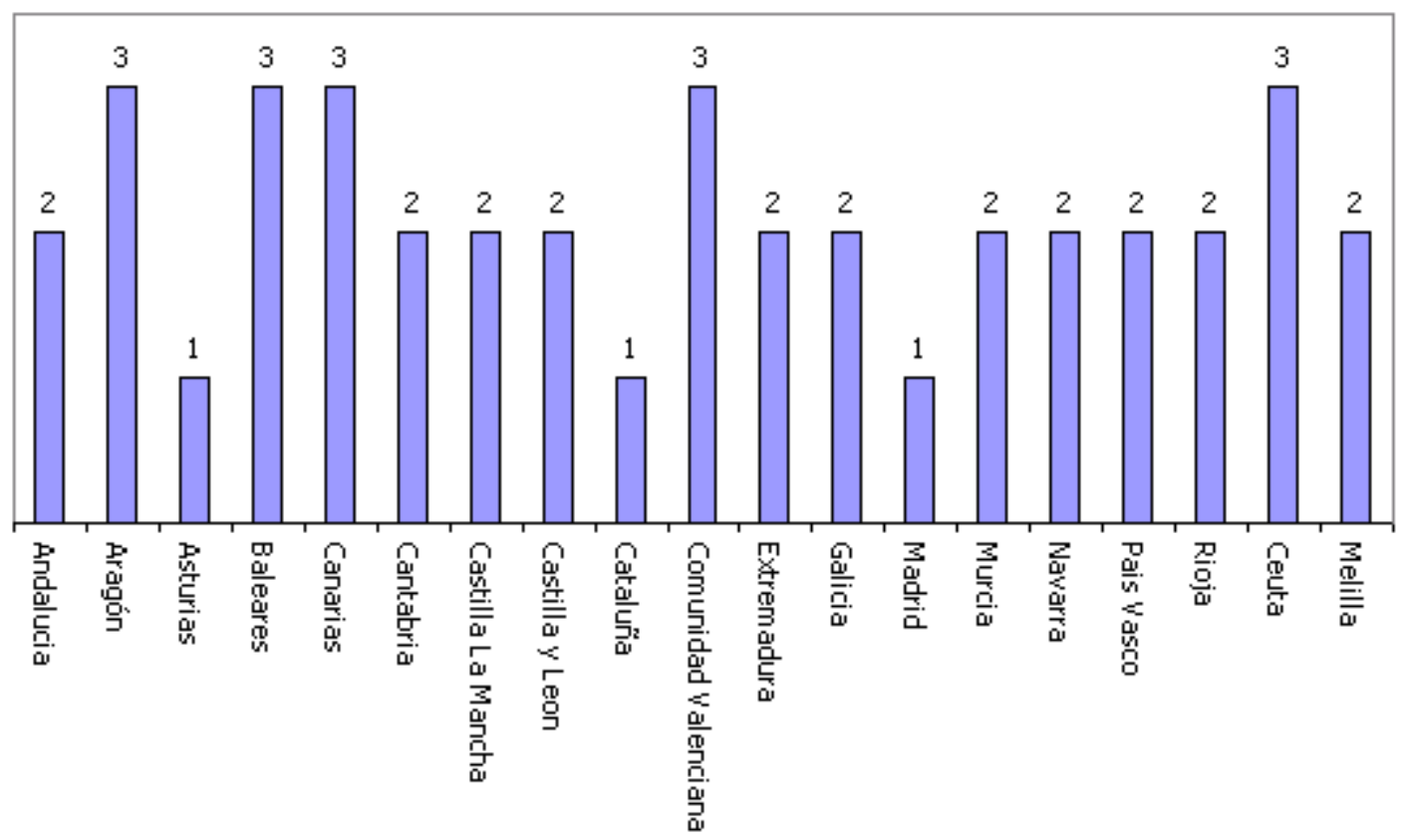

* Fuente: Elaboración propia (donde 1 es el mejor resultado y 3 el peor).

\subsection{Tratamiento de los contenidos}

A pesar de que los contenidos en muchas ocasiones están ligados al diseño del soporte, en este caso pretendemos hacer un repaso a los ofertados en las webs institucionales sin tomar en consideración su organización, un aspecto que será evaluado en el siguiente apartado.

En este caso, abordamos aquella información mínima que deberían contemplar las webs de las Comunidades Autónomas. Entre estos aspectos, destacamos elementos institucionales, notas de prensa, imágenes, agenda de actividades, encuestas, diferentes niveles de información, boletín de novedades e información de los órganos de Gobierno. Todo ello también analizado en función de la forma de ofrecerse al público. Con este planteamiento podemos distinguir variaciones entre las propuestas y se plantean de nuevo tres bloques en relación a su adecuación.

En el primero, observamos a Catalunya (www.gencat.net), La Rioja (www.larioja.org), Comunidad de Madrid (www.madrid.org), Principado de Asturias (www.asturias.es), Andalucía (www.andaluciajunta.es), Extremadura (www.juntaex.es), Navarra (www.navarra.es), Región de Murcia (www.carm.es), Castilla La Mancha (www.jccm.es) y País Vasco (www.euskadi.net). Estas páginas se caracterizan por su facilidad de navegación y con unos contenidos claros y sencillos orientados al ciudadano. Por decirlo de alguna forma, son páginas resolutivas. En su mayoría, complementan la información necesaria para la realización de trámites, tanto físicamente como 
telemáticamente bien para la propia Administración autónoma como para otras Instituciones. Además, todos estos portales, en mayor o menos medida, permiten una comunicación bidireccional entre ciudadanos y Administraciones. Por lo que respecta al contenido informativo, hay que destacar el caso de la Comunidad de Madrid que incluye numerosos complementos de apoyo que acompañan a las notas de prensa (fotografía, audio y vídeo). De la Generalitat de Catalunya hay que valorar la variedad de boletines informativos que ofrece a través del móvil, RSS o multimedia. Por otro lado, hay que indicar la oferta de contenidos noticiosos que aporta la web de Extremadura a los que se puede acceder desde el portal de inicio en un enlace denominado “Junta al día”, con versión para pda, wap e incluso con cobertura en directo de algunos acontecimientos. Otra web a destacar es la de Castilla La Mancha que incorpora boletines RSS, agenda de eventos, fotografías en alta resolución y noticias leidas.

En el segundo bloque observamos a Melilla (www.melilla.es), Aragón (www.aragon.es), Cantabria (www.gobcantabria.es), Castilla León (www.jcyl.es), Galicia (www.xunta.es), Baleares (www.caib.es), Comunidad Valenciana (www.gva.es), Ceuta (www.ceuta.es) y Canarias (www.gobcan.es). Contienen un volumen importante de información para desarrollar trámites con la propia autonomía, pero no ofrecen la misma cantidad de información relativa a las gestiones que se deben llevar a cabo con otras Instituciones, un aspecto que sí se halla en las del primer grupo. A su vez, la mayoría presenta también una fácil navegación, sin demasiadas complicaciones para el usuario a la hora de encontrar los contenidos que busca. En cuanto a los elementos noticiosos, hay que valorar el caso de la Región de Murcia porque cuenta con un espacio del área de comunicación en el portal de inicio que ofrece varios enlaces que permiten, entre otros, descargar las previsiones informativas, los acuerdos del Consejo de Gobierno, notas de prensa, agenda de la comunicación o archivos multimedia relacionados con los eventos. Otro ejemplo a valorar es el de Andalucía ya que encontramos un aviso en la página de inicio en el que se indica que las noticias pueden ser consultadas en castellano y lengua de signos española y audiodescritas.

En este apartado no se ha clasificado a ninguna página en el tercer apartado ya que todas ellas presentan en mayor o menor grado información tanto institucional como de actualidad.

\section{Gráfico 2: Tratamiento de los contenidos}




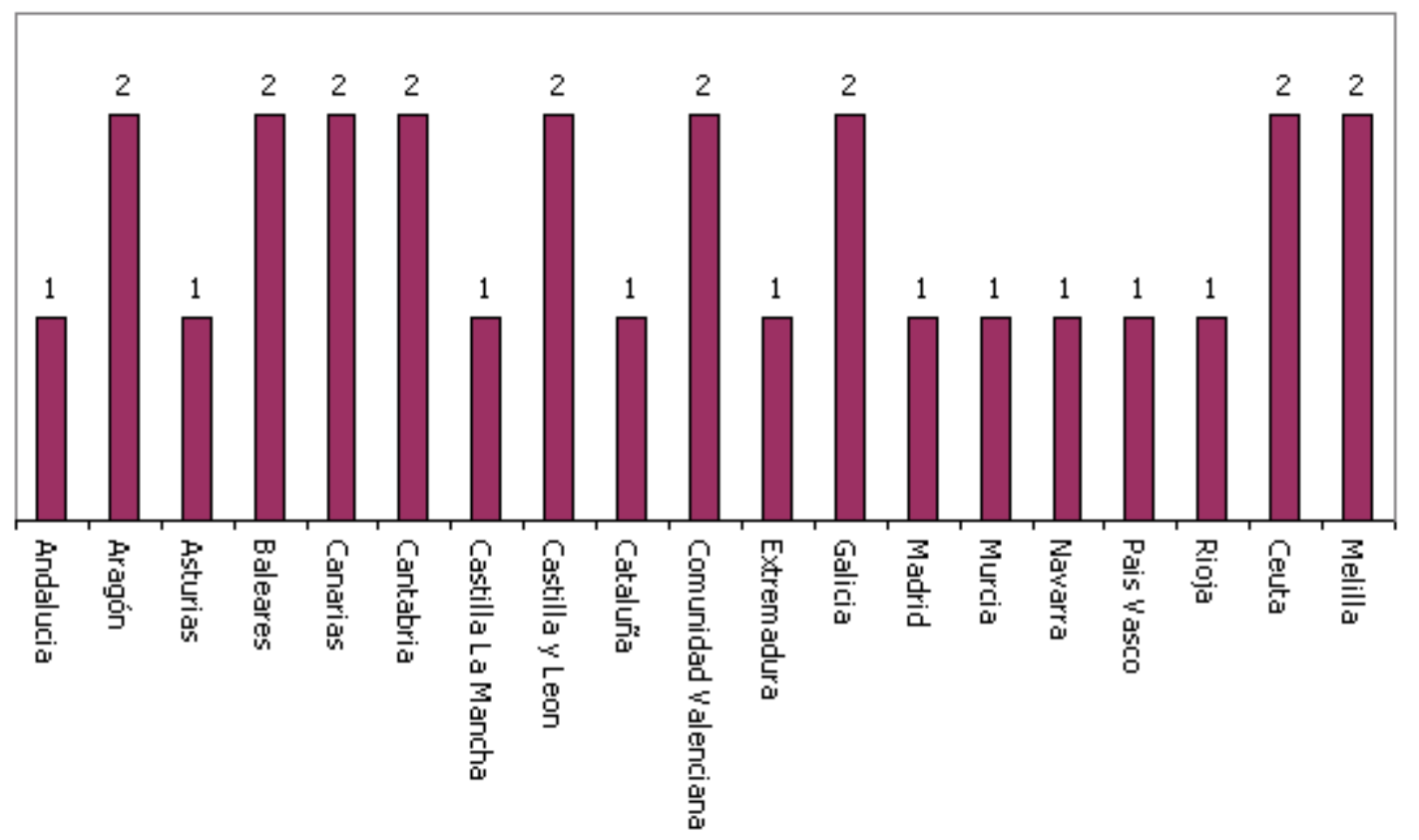

* Fuente: Elaboración propia (donde 1 es el mejor resultado y 3 el peor).

\subsection{Imagen y diseño}

La identificación del ciudadano con la imagen corporativa ofrecida a través de la web es uno de los elementos esenciales de cara a incorporar estos portales como un elemento más dentro de la planificación global de la comunicación institucional. El diseño planteado en cada una de las propuestas abordadas está estrechamente relacionado con la imagen proyectada en primer lugar de la web, pero en el fondo de la Institución, sobre todo teniendo en cuenta que el portal debe ser la ventana de acceso de los ciudadanos. Un planteamiento que todavía queda reforzado cuando estamos hablando de e-Administración y prestación de servicios públicos a los ciudadanos de forma telemática.

Las Instituciones no deben solamente cuidar la imagen de los ámbitos donde prestan sus servicios de forma física sino también la virtual que con la Ley 11/2007, de 22 de junio, de acceso electrónico de los ciudadanos a los Servicios Públicos cobra la misma validez.

Es pues uno de los elementos fundamentales a la hora de hacer un planteamiento global de comunicación que, aunque cada vez es asumido de una forma más clara en las Administraciones Públicas, todavía existen casos en los que la imagen proyectada no es la ideal en función de los 
servicios prestados. De hecho, como veremos, hay ejemplos en los que se puede llegar incluso a distorsionar la imagen institucional.

Esta imagen proyectada está ligada de forma estrecha a los dos aspectos abordados anteriormente, al de la facilidad o no de disponer de los servicios públicos de forma telemática y a los contenidos incorporados. De todos modos, sin un buen trabajo en las dos materias anteriormente citadas el diseño pierde importancia. Dividiremos las webs analizadas en tres grupos:

El primero, en el que situamos a Castilla León (www.jcyl.es), Asturias (www.asturias.es) y Murcia (www.carm.es), se caracteriza por el buen uso de la tipografía escogida, los colores utilizados, la distribución de los contenidos, la accesibilidad y traducción en diferentes idiomas, entre otros aspectos.

El segundo de los bloques lo conforman las webs de Cataluña (www.gencat.net), Madrid (www.madrid.org), Andalucía (www.andaluciajunta.es), Baleares (www.caib.es), Canarias (www.gobcan.es), La Rioja (www.larioja.org), Navarra (www.navarra.es), País Vasco (www.euskadi.net), Galicia (www.xunta.es), Cantabria (www.gobcantabria.es), Melilla (www.melilla.es), Aragón (www.aragon.es), Extremadura (www.juntaex.es) y Ceuta (www.ceuta.es). Estos portales se diferencian del grupo anterior aunque las tipografías y los colores utilizados son correctos para la visualización el diseño estructural planteado no mantiene tan buena sintonía con la imagen corporativa de la Institución como las del bloque anterior. De este conjunto, hay que hacer una referencia a la web de la Ciudad Autónoma de Ceuta que aunque tiene un diseño atractivo se trata de una página muy lenta en la navegación lo que dificulta el acceso a la información y ralentiza las consultas de los usuarios. En esta misma categoría, también hay que valorar en la página de la Generalitat de Cataluña, que a pesar de ofrecer unos servicios excelentes y unos contenidos muy completos, el acceso al portal de entrada puede resultar en algunos casos desconcertante para el usuario debido al gran volumen de información que se ofrece.

El tercer grupo lo forman Castilla La Mancha (www.jccm.es) y la Comunidad Valenciana (www.gva.es). En la primera de ellas, el excesivo uso de imágenes tanto dinámicas como estáticas a modo de banners hace que el ciudadano pierda la perspectiva de la información que está buscando. Además, los colores utilizados en el apartado del portal de entrada que se denomina "Máximo interés” dificultan la consulta e incluso hasta la propia lectura. También los estilos usados en la tipografía no son los más adecuados. Por su parte, en la web de la Comunidad Valenciana existe un cierto abuso de la utilización de las imágenes en el portal de inicio, una gran cantidad de información 
que dificulta al usuario, tal y como está planteada, su consulta. Además, cuenta con un menú dinámico que se desplaza horizontalmente en la parte media-superior de la página que incomoda la visualización.

\section{Gráfico 3: Imagen y diseño}

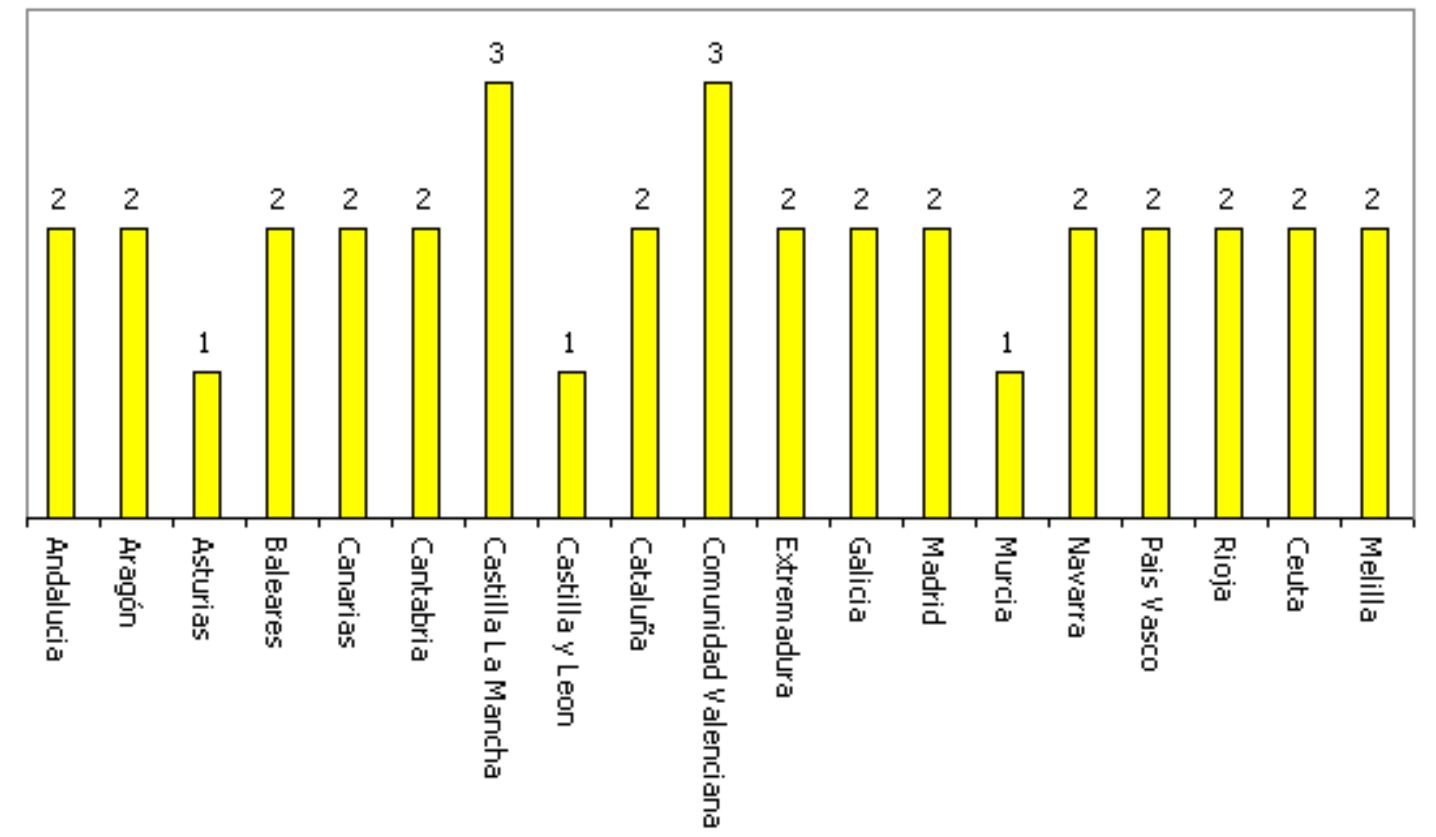

* Fuente: Elaboración propia (donde 1 es el mejor resultado y 3 el peor).

\section{Conclusiones}

- Las páginas webs de las administraciones públicas han de ser la nueva puerta de entrada de los ciudadanos a los servicios que estas prestan. Por tanto, tienen que ofrecer las mismas posibilidades a los usuarios que tienen en las ventanillas físicas que existen.

- $\quad$ Los contenidos ofrecidos, la forma en que son aportados, el diseño y la imagen corporativa son elementos claves para conseguir una identificación del ciudadano y para ganarse su confianza con el fin de que asuman la necesidad de utilización de las herramientas telemáticas de cara a que se mejore la gestión pública en términos de eficacia, eficiencia y rapidez.

- $\quad$ El estudio que se ha realizado sobre las 19 webs, entre Comunidades Autónomas y Ciudades Autónomas, revela que únicamente una, la de Asturias (www.asturias.es), se sitúa en las tres 
categorías dentro del grupo más valorado. Esta página cumple tanto en los servicios públicos ofrecidos, en la forma de tratamiento de los contenidos y en la imagen y el diseño realizado con los parámetros estudiados en el trabajo.

En el lado opuesto, observamos a la Comunidad Valenciana (www.gva.es), que cuenta con el único portal de los 19 abordados que se sitúa en dos de las tres categorías analizadas dentro del bloque que menos cumple con los objetivos determinados.

- $\quad$ En la zona media-alta de valoración observamos a Andalucía (www.andaluciajunta.es), Cantabria (www.gobcantabria.es), Castilla y León (www.jcyl.es), Cataluña (www.gencat.net), Extremadura (www.juntaex.es), Galicia (www.xunta.es), Madrid (www.madrid.org), Murcia (www.carm.es), Navarra (www.navarra.es), País Vasco (www.euskadi.net), La Rioja (www.larioja.org) y Melilla (www.melilla.es). Ninguno de estos portales ha sido valorado de forma negativa en alguna de las tres categorías, un aspecto que evidencia el trabajo realizado por sus Administraciones.

- $\quad$ En la zona media-baja se observa a Baleares (www.caib.es), Aragón (www.aragon.es),

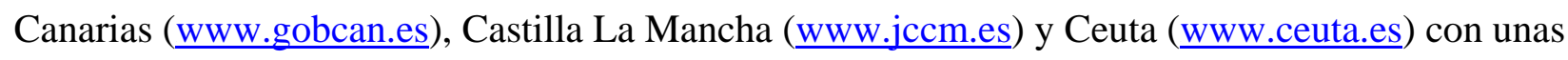
páginas en la que una de las tres categorías ha sido calificada como de negativa al no cumplir con los mínimos planteados.

\section{Gráfico 4: Comparativa de las tres categorías analizadas}

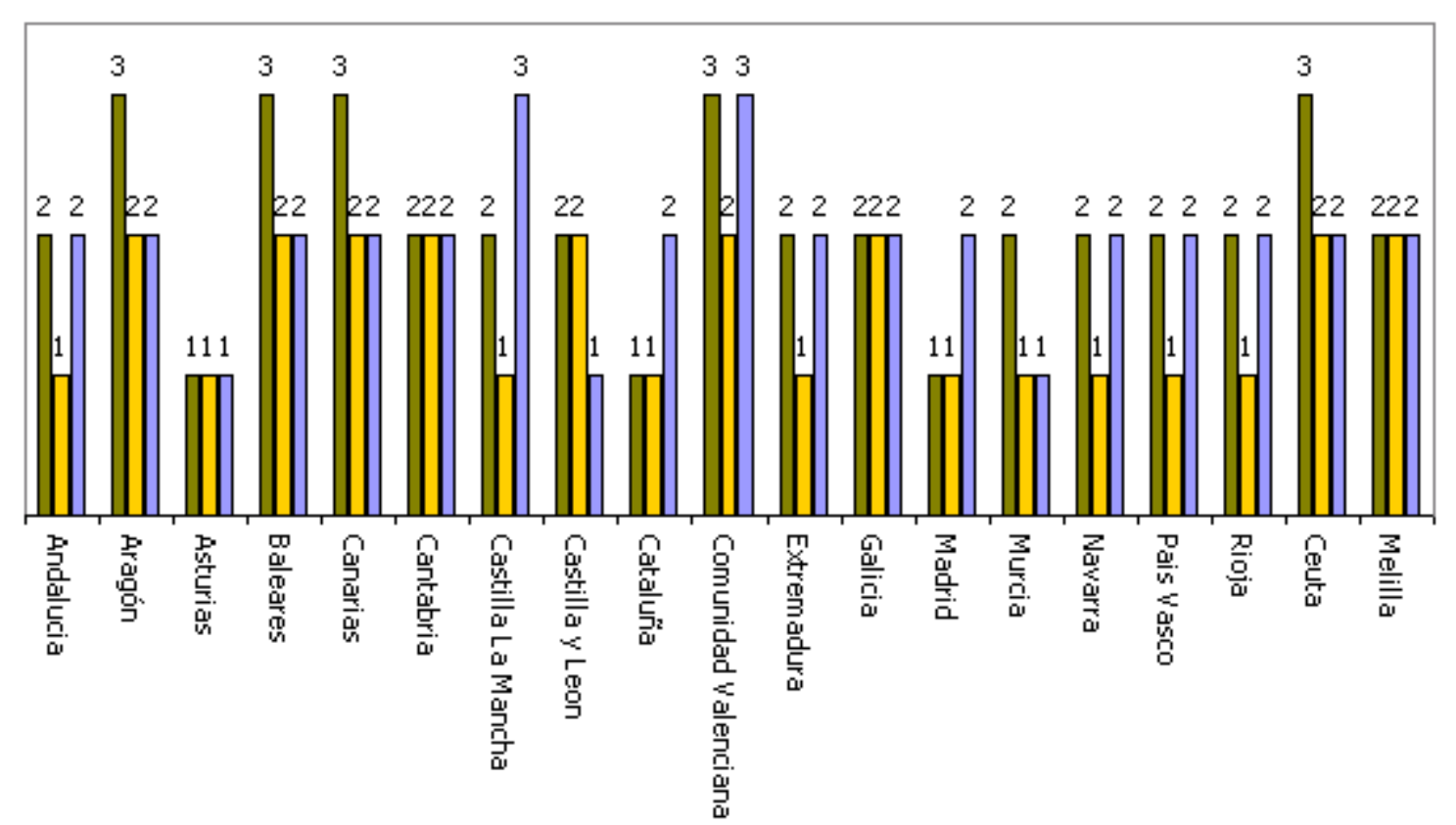


* Fuente: Elaboración propia (donde 1 es el mejor resultado y 3 el peor).

- $\quad$ Uno de los elementos a destacar dentro de este proceso es el presupuesto TIC/habitante en cada una de las Comunidades Autónomas. En este sentido, hay que destacar que el País Vasco y el Principado de Asturias son las comunidades que obtienen un resultado más elevado (87,46 y 61,47 euros), coincidiendo con dos de las autonomías mejor valoradas en el trabajo. La media en el total de España es de 33,33, según datos del informe de la Fundación AUNA (2005).

El presupuesto TIC/habitante ( $€$ per capita) en el resto de Comunidades Autónomas es el siguiente: Andalucía (31,29), Aragón (21,48), Baleares (14,61), Canarias (48,29), Cantabria $(23,81)$, Castilla La Mancha (34,27), Castilla León (28,07), Cataluña (29,5), Comunidad Valencia $(30,78)$, Extremadura (25,48), Galicia (17,79), Comunidad de Madrid (30,32), Región de Murcia $(10,21)$, Navarra $(35,23)$ y La Rioja (31).

\section{Gráfico 5: Presupuesto TIC/habitante (€ per capita)}

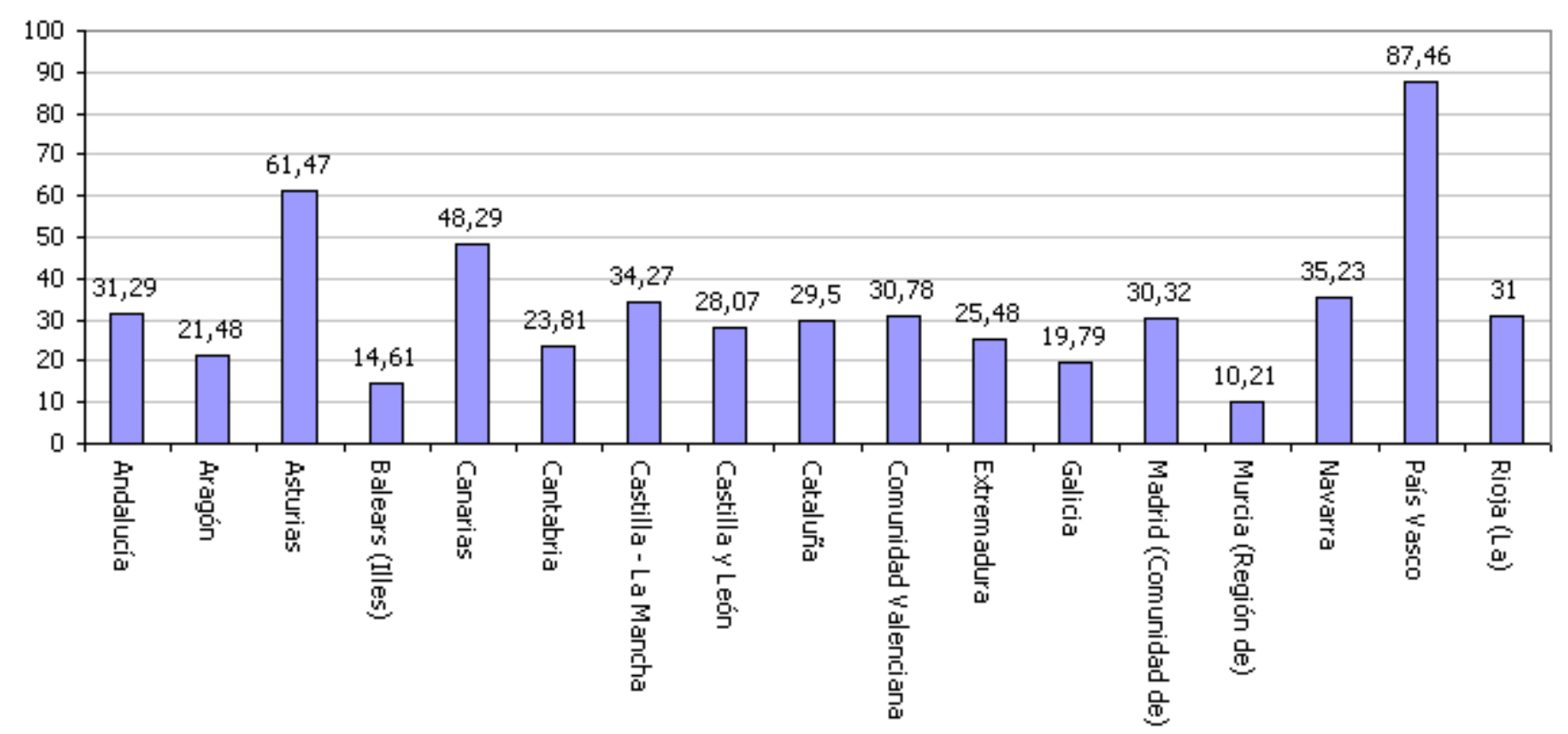

* Elaboración propia. Fuente: Fundación AUNA (2005) 


\section{Bibliografía}

- ANGULO MARTÍN, C. I., Ciencia, tecnología y sociedad, Instituto Nacional de Estadística (INE), Madrid. (Informe elaborado por el Jefe de Área de Indicadores e Informes Sociales del INE).

- BERELSON, B., 1971, Content analisis in communication research, Hafner, Nueva York.

- FUNDACIÓN AUNA, 2005, eEspaña 2005, Fundación Auna, Madrid.

- FUNDACIÓN ORANGE, 2007, eEspaña 2007. Informe anual sobre el desarrollo de la sociedad de la información en España, Fundación Orange, Madrid.

- OLSEN, L., RIEPER, O. y TORPE, L., 2006, “E-participation in local governance in Denmarrk” en HANSEN, H. K. y HOFF, J., 2006, Digital Governance://Networked Societes. Creating Authority, Community and Identity in a Globalized World. Nordicom, Frederiksberg.

- HERNÁNDEZ SAMPIERI, R.; FERNANDEZ COLLADO, C.; BAPTISTA LUCIO, P., 2004, Metodología de la Investigación, McGrawHill, Santiago de Chile.

- IGARTUA, J. J., 2006, Métodos Cuantitativos de investigación en comunicación, Bosch Comunicación, Barcelona.

- KRIPPENDORFF, K., 1990, Metodología de análisis de contenido. Teoría y práctica, Paidós Comunicación, Barcelona.

- MANCHÓN, E., 2002, ¿Qué es la usabilidad? en ainda.info, visitado el día 3/12/2007, http://www.ainda.info/que_es_usabilidad.htm

- MURCIANO, M.; SABÉS, F. y otros, 2007, Nueva tecnología de la información y cambio comunicativo en las Comunidades Autónomas Españolas (proyecto de investigación SEC 200307024). Pendiente de publicación.

NIELSEN, J., 2000, Usabilidad. Diseño de sitios web, Prentice Hall, Madrid.

-PARDO KUKLINSKI, H., 2006, Pautas hacia un modelo de aplicación web institucional universitaria. El caso de los webcom: sitios de facultades de comunicación de Iberoamérica en ZER, $n^{\circ}$ 21, Universidad del País Vasco.

- SABÉS TURMO, F., 2007, La Ley 11/2007, de 22 de junio, de acceso electrónico de los ciudadanos a los Servicios Públicos, un importante paso para la eAdministración en España en Signo y Pensamiento, ${ }^{\circ}$ 51, Universidad Javeriana de Bogotá. 
- TORREJÓN, A.M. La Nueva Geografía y las cifras de la Sociedad de la Información, en Fundación Orange, visitado el día 4/1/2008.

http://www.fundacionorange.es/areas/28_observatorio/obser_01_13.asp 